\title{
Using bivariate signal analysis to characterize the epileptic focus: The benefit of surrogates
}

\author{
R. G. Andrzejak, ${ }^{1, *}$ D. Chicharro, ${ }^{1}$ K. Lehnertz, ${ }^{2,3,4}$ and F. Mormann ${ }^{2}$ \\ ${ }^{1}$ Department of Information and Communication Technologies, Universitat Pompeu Fabra, Barcelona, Spain \\ ${ }^{2}$ Department of Epileptology, University of Bonn, Bonn, Germany \\ ${ }^{3}$ Helmholtz-Institute for Radiation and Nuclear Physics, University of Bonn, Bonn, Germany \\ ${ }^{4}$ Interdisciplinary Center for Complex Systems, University of Bonn, Bonn, Germany \\ (Received 13 October 2010; revised manuscript received 27 January 2011; published 7 April 2011)
}

\begin{abstract}
The disease epilepsy is related to hypersynchronous activity of networks of neurons. While acute epileptic seizures are the most extreme manifestation of this hypersynchronous activity, an elevated level of interdependence of neuronal dynamics is thought to persist also during the seizure-free interval. In multichannel recordings from brain areas involved in the epileptic process, this interdependence can be reflected in an increased linear cross correlation but also in signal properties of higher order. Bivariate time series analysis comprises a variety of approaches, each with different degrees of sensitivity and specificity for interdependencies reflected in loweror higher-order properties of pairs of simultaneously recorded signals. Here we investigate which approach is best suited to detect putatively elevated interdependence levels in signals recorded from brain areas involved in the epileptic process. For this purpose, we use the linear cross correlation that is sensitive to lower-order signatures of interdependence, a nonlinear interdependence measure that integrates both lower- and higher-order properties, and a surrogate-corrected nonlinear interdependence measure that aims to specifically characterize higher-order properties. We analyze intracranial electroencephalographic recordings of the seizure-free interval from 29 patients with an epileptic focus located in the medial temporal lobe. Our results show that all three approaches detect higher levels of interdependence for signals recorded from the brain hemisphere containing the epileptic focus as compared to signals recorded from the opposite hemisphere. For the linear cross correlation, however, these differences are not significant. For the nonlinear interdependence measure, results are significant but only of moderate accuracy with regard to the discriminative power for the focal and nonfocal hemispheres. The highest significance and accuracy is obtained for the surrogate-corrected nonlinear interdependence measure.
\end{abstract}

DOI: 10.1103/PhysRevE.83.046203

PACS number(s): 05.45.Tp, 05.45.Xt, 87.19.1e, 87.19.1m

\section{INTRODUCTION}

A characterization of interactions between dynamical systems from the analysis of signals recorded from them is key to an understanding of many phenomena studied in natural and social sciences. A paradigmatic example for this setting is the brain and the study of electrophysiological recordings derived from it. Complex brain functions are based on neuronal interactions at a variety of temporal and spatial scales, and neurological disorders often involve distorted balances in these interactions. In epilepsy, distorted neuronal interactions are assumed to result in recurrent acute dysfunctions of the brain that manifest themselves as seizures. During epileptic seizures, large groups of neurons discharge hypersynchronously. In consequence, the electroencephalogram (EEG) recorded during epileptic seizures is characterized by rhythmic oscillations of high amplitude. During the seizurefree interval, only intermittent bursts of hypersynchronous activity of local neuron groups occur, resulting in so-called interictal epileptiform waveforms in the EEG. Except for these episodes of interictal epileptiform activity, which can last tens of milliseconds to seconds, the EEG recorded during the seizure-free interval often appears unspecific with regard to the epileptic process. It can be conjectured, however, that even in the absence of evident interictal epileptiform activity, the epileptic process is associated with an elevated level of

*ralph.andrzejak@upf.edu neuronal synchronization. In the case of focal epilepsies, this synchronization can furthermore be conjectured to be most pronounced in the seizure generating area, the so-called epileptic focus.

To test these conjectures, a number of conceptually different bivariate signal analysis techniques were applied to intracranial EEG recordings from the seizure-free interval of epilepsy patients in order to characterize interactions between brain areas underlying different recording sites. These approaches include linear coherence [1,2], linear cross correlation [3], genuine linear cross correlation [4], a nonlinear correlation coefficient [5], nonlinear interdependence measures [3,6], Hilbert phase synchronization $[3,7,8]$, or event phase synchronization [9]. Results of these studies provide converging evidence that the overall interdependence between EEG signals recorded close to the epileptic focus is higher than in those recorded from nonepileptogenic brain regions. Furthermore, the predominant direction of information flow and interdependencies between the epileptic focus and nonepileptogenic brain regions was studied using information theoretic measures [10-12] or measures derived from estimated Fokker-Planck coefficients [13]. Together, these studies [1-13] suggest that bivariate signal analysis techniques could be used to localize the epileptic focus and thereby provide useful diagnostic information.

To provide further progress in this direction, we compare here the accuracy of three different approaches: bivariate linear time series analysis, bivariate nonlinear time series analysis, and surrogate-corrected bivariate nonlinear time series analysis. Linear time series analysis allows quantifying 
lower-order properties of time series. For example, univariate and bivariate linear time series analysis measures are based on autocorrelation and cross-correlation functions. Nonlinear time series analysis has been developed to study higher-order structure, which is characteristic for nonlinear deterministic dynamics. Univariate nonlinear measures allow quantifying properties of individual deterministic dynamics such as the number of active degrees of freedom. Bivariate nonlinear measures allow detecting signatures indicative of interdependence between deterministic dynamics. Both univariate and bivariate nonlinear measures are, however, strongly influenced by linear correlations of the signals. Deterministic dynamics typically show some degree of autocorrelation, and a coupling between deterministic dynamics manifests itself not only in higher-order signatures of interdependence, but also in a strong cross correlation. Likewise, however, linear stochastic processes can be strongly autocorrelated, and arbitrary degrees of cross correlation can be obtained for multivariate linear stochastic processes or from superpositions of completely independent signals originating from any type of dynamics. Hence, nonlinear time series analysis measures are sensitive for properties of nonlinear deterministic dynamics without being very specific.

This lack of specificity can be addressed with the Monte Carlo concept of surrogates [14], first origins of which can be found in the literature on electrocardiogram [15] and electroencephalogram [16] analysis. Surrogates allow testing a variety of well-defined null hypotheses about the dynamics underlying experimental signals. For this purpose, surrogate time series can be generated from a constrained randomization of the original time series. Therefore, only well-specified properties of the original time series, such as the autocorrelation and cross correlation, are mimicked by the surrogates. Otherwise, the surrogates are random. It depends on the particular null hypothesis to be tested as to which properties have to be preserved and which have to be randomized $[14,17,18]$. Comparing values of nonlinear time series analysis measures calculated for the original time series against a distribution of values calculated from an ensemble of surrogate times series allows one to test the surrogates' null hypothesis.

Beyond a binary decision derived from formal null hypothesis testing, surrogates can be used to define a baseline for nonlinear measures expected under the null hypothesis. For the case of univariate time series analysis, we showed that this surrogate correction improves the accuracy in characterizing the spatial distribution of the epileptic process [19] (see also [20,21] and references therein for applications of different univariate time series analysis approaches to the EEG of epilepsy patients). This previous study compared univariate linear measures, univariate nonlinear measures, and univariate surrogate-corrected nonlinear measures in application to intracranial EEG recordings from the seizurefree interval of 29 patients with an epileptic focus located in the medial temporal lobe. The highest accuracy in determining the focal hemisphere was obtained for the surrogate-corrected nonlinear measures, while the discriminative power of the other two approaches was weaker if not insignificant.
We study here whether these findings carry over from univariate to bivariate nonlinear time series analysis. For this purpose, we analyze the same EEG recordings that we previously studied using univariate measures in [19]. We combine a nonlinear interdependence measure with bivariate surrogates and contrast the results from this surrogate-corrected measure to those obtained from the linear cross correlation and the nonlinear interdependence measure without surrogate correction. In analogy to [19], we determine the suitability of these different approaches to determine the brain hemisphere containing the epileptic focus.

\section{METHODS}

\section{A. Bivariate time series analysis measures}

\section{Linear cross correlation}

We suppose that a pair of scalar time series $x_{n}$ and $y_{n}$, with $n=0, \ldots, N-1$, were simultaneously recorded from the dynamics $X$ and $Y$, respectively. The cross correlation quantifies the strength of the linear correlation between these two time series' amplitudes as a function of a temporal shift $\tau$ between them:

$$
C_{x y}(\tau)=\left\{\begin{array}{cc}
\frac{1}{N-\tau} \sum_{n=0}^{N-\tau-1} \hat{x}_{n+\tau} \hat{y}_{n} & \tau \geqslant 0, \\
C_{y x}(-\tau) & \tau<0
\end{array}\right.
$$

for $\tau=-(N-1), \ldots, N-1$. In Eq. (1), $\hat{x}_{n}, \hat{y}_{n}$ denote the time series $x_{n}, y_{n}$ normalized to zero mean and unit variance. As a linear bivariate measure, we use

$$
C=\left|C_{x y}(\tau=0)\right|,
$$

where $|\cdots|$ denotes the absolute value. [We also tested an alternative measure $C^{*}=\max \left\{\left|C_{x y}(\tau)\right|\right\}_{\tau=-\tau_{\max }, \ldots, \tau_{\max }}$. For this measure, we limited the range of temporal shifts to $\tau_{\max }=2 \mathrm{~s}$, because the number of summands contributing to $C_{x y}(\tau)$ is $N-\tau$, resulting in strong fluctuations of $C_{x y}(\tau)$ for $\tau \rightarrow N$. Since the final averaged results derived from $C$ and $C^{*}$ were very similar, and since the nonlinear interdependence measure was also calculated only for zero temporal shift between the time series, we here only show results of $C$.]

\section{Nonlinear interdependence measure}

A nonsynchronizing directional coupling between two stationary deterministic dynamics $X$ and $Y$ results in a characteristic signature of interdependence [6,22-24]. Consider a set of similar states in the $Y$ dynamics. These similar states will be close in some state space representation of $Y$. If $X$ is driving $Y$, the states in $X$ that are simultaneous to similar states in $Y$ are on average closer than expected for independent dynamics. The opposite mapping, i.e., closeness in $X$ implying closeness in $Y$, generally holds only to a weaker degree. Various nonlinear interdependence measures [6,18,22-28] quantify this fundamental signature in order to detect directional couplings.

For this paper, we use a nonlinear interdependence measure $L$, which was shown to be of higher sensitivity and specificity 
for directional couplings than a number of related approaches [28]. For the calculation of $L$, we first reconstruct the dynamics $X$ and $Y$ from the pair of univariate time series $x_{n}$ and $y_{n}$ using delay coordinates [29]

$$
\begin{aligned}
& \mathbf{x}_{n}=\left(x_{n}, x_{n-\tau_{D}}, \ldots, x_{n-(m-1) \tau_{D}}\right), \\
& \mathbf{y}_{n}=\left(y_{n}, y_{n-\tau_{D}}, \ldots, y_{n-(m-1) \tau_{D}}\right)
\end{aligned}
$$

for $n=(m-1) \tau_{D}, \ldots, N-1$. Here $m$ and $\tau_{D}$ denote the embedding dimension and embedding delay, respectively. We then calculate the squared Euclidean spatial distances $d\left(\mathbf{x}_{i}, \mathbf{x}_{j}\right)$ and $d\left(\mathbf{y}_{i}, \mathbf{y}_{j}\right)$ for all $i, j=(m-1) \tau_{D}, \ldots, N-1$. Here we apply a Theiler window by imposing $|i-j|>W$ [30]. To test for directional couplings from $X$ to $Y$, we carry out the following steps. For each fixed $i_{0}, j_{0}$, we use $g\left(\mathbf{x}_{i_{0}}, \mathbf{x}_{j_{0}}\right)$ to denote which rank the distance $d\left(\mathbf{x}_{i_{0}}, \mathbf{x}_{j_{0}}\right)$ has in a sorted ascending list of all $N-(m-1) \tau_{D}-1$ distances $d\left(\mathbf{x}_{i_{0}}, \mathbf{x}_{j}\right)$. Furthermore, for each fixed $i_{0}$, the $j$ indices of the $\kappa$ smallest of all $N-(m-1) \tau_{D}-1$ distances $d\left(\mathbf{y}_{i_{0}}, \mathbf{y}_{j}\right)$ are denoted by $j_{i_{0}, w}$ for $w=1, \ldots, \kappa$. We define the $Y$-conditioned mean rank

$$
G_{i_{0}}^{\kappa}(X \mid Y)=\frac{1}{\kappa} \sum_{w=1}^{\kappa} g\left(\mathbf{x}_{i_{0}}, \mathbf{x}_{j_{i_{0}, w}}\right)
$$

and carry out a normalized statistics across all embedding vectors

$$
L(X \mid Y)=\frac{1}{N-(m-1) \tau_{D}} \sum_{i=(m-1) \tau_{D}}^{N-1} \frac{G_{i}(X)-G_{i}^{\kappa}(X \mid Y)}{G_{i}(X)-G_{i}^{\kappa}(X)},
$$

where $G_{i}(X)=\frac{N-(m-1) \tau_{D}}{2}$ and $G_{i}^{\kappa}(X)=\frac{\kappa+1}{2}$ denote the mean rank and minimal mean rank, respectively [28]. [Due to the exclusion of temporal neighbors within a Theiler window of length $W$, the numerator in the formula for $G_{i}(X)$ needs to be adjusted, and cases $i<W+(m-1) \tau_{D}, i \geqslant N-W+1$ need to be further distinguished. To simplify the notation, here we write it for $W=0$. The source code to calculate $L(X \mid Y)$ based on the adjusted formulas is provided as supplementary material of [28].]

For independent dynamics, we obtain $G_{i}^{\kappa}(X \mid Y) \approx G_{i}(X)$, and values of $L(X \mid Y)$ are distributed around zero for independent realizations of independent dynamics. For directional couplings from $X$ to $Y$ states that are close for the driven dynamics $Y$ correspond more often to close contemporary states in the driving dynamics $X$ than expected for independent dynamics. In consequence, $G_{i}^{\kappa}(X \mid Y)<G_{i}(X)$ and positive values are obtained for $L(X \mid Y)$. Increasing coupling strengths are generally reflected in increasing values of $L(X \mid Y)$. The upper bound is obtained for identical synchronization, where $X=Y$ and we obtain $G_{i}^{\kappa}(X \mid Y)=G_{i}^{\kappa}(X)$ and $L(X \mid Y)=1$. To test for directional couplings from $Y$ to $X$, we calculate $L(Y \mid X)$ in the same way as $L(X \mid Y)$ by exchanging the roles of $X$ and $Y$ in the above definitions. The difference $L(X \mid Y)-L(Y \mid X)$ has an expected value of zero for independent dynamics, and nonzero differences can be used to characterize the direction of couplings [28]. Since here we focus on the overall strength rather than on the direction of the coupling, we define
$[31,32]$

$$
L=\frac{L(X \mid Y)+L(Y \mid X)}{2} .
$$

The measure $L$ assumes values distributed around zero for independent realizations of independent dynamics, and its upper bound is $L=1$ for identical synchronization. Increasing coupling strengths are generally reflected in increasing values of $L$.

\section{Surrogate time series}

We use bivariate surrogates constructed using an iterative scheme proposed by Schreiber and Schmitz [17]. This particular type of bivariate surrogates is designed to test the null hypothesis $\mathcal{H}_{0 \text {,biv }}: X$ and $Y$ jointly represent a stationary bivariate linear stochastic correlated Gaussian process. The measurement functions by which $x_{n}$ and $y_{n}$ were derived from the dynamics are invertible but potentially nonlinear. The autocorrelation, cross correlation, mean, and variance of the underlying Gaussian process are such that the measurement results in the autocorrelation, cross correlations, and amplitude distribution of the observed time series. Accordingly, any pair of surrogate time series should have the same autocorrelation and cross correlation as the original pair of time series. Furthermore, to account for a potential nonlinearity of the measurement function, the surrogates should have the same amplitude distribution as the original time series. However, any potential nonlinear deterministic structure, nonstationary features, or potential signatures of nonlinear interdependence in $x_{n}$ and $y_{n}$ should be destroyed. Bivariate surrogates that fulfill these constraints were generated using the scheme described in Appendix A.

\section{Surrogate-corrected nonlinear interdependence measure}

For each pair of time series, we calculate $L$ for the original time series $x_{n}$ and $y_{n}$ and for an ensemble of 19 independent realizations of pairs of surrogate time series $\widetilde{x}_{n}$ and $\widetilde{y}_{n}$. We denote the value obtained for the pair of original time series by $L_{O}$ and the mean value obtained across all 19 pairs of surrogate time series by $\left\langle L_{S}\right\rangle$. From this, we define

$$
K=\left\{\begin{array}{ccc}
L_{O}-\left\langle L_{S}\right\rangle & \text { if } & L_{O}-\left\langle L_{S}\right\rangle>0 \\
0 & \text { otherwise }
\end{array}\right.
$$

\section{B. Patients and electroencephalographic recordings}

As stated in the Introduction, we analyze here the same set of intracranial EEG recordings that were analyzed in [19]. These recordings were performed as part of the presurgical epilepsy diagnostics (Department of Epileptology, University of Bonn, Germany) in 29 patients who were refractory to medical therapy. All patients were diagnosed with unilateral medial temporal lobe epilepsy (left hemisphere: 18 patients; right hemisphere: 11 patients). In consequence, all patients underwent surgery (selective amygdalohippocampectomy), which led to complete seizure control in all patients as 


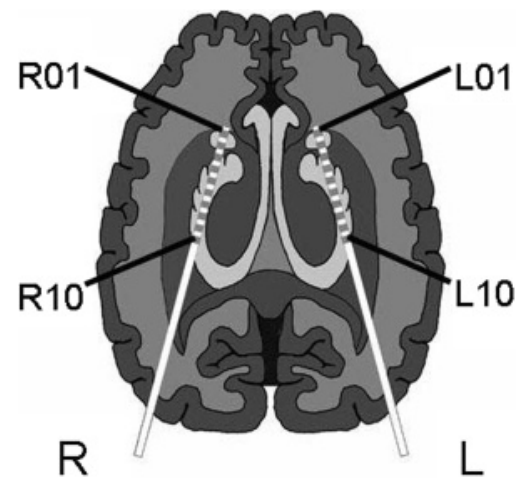

FIG. 1. Scheme of implanted electrodes.

documented for at least 1 year after surgery (mean: 3.8 years, range: $1-9$ years).

The EEG was recorded using pairs of depth electrodes that were implanted symmetrically in the hippocampal formations of the two hemispheres (Fig. 1). Electrodes were each equipped with 10 cylindrical contacts of $2.5 \mathrm{~mm}$ length and an intercontact distance of $4 \mathrm{~mm}$. After neurosurgical implantation, the placement of the electrodes was verified by magnetic resonance imaging. The EEG was recorded against a common average reference at a sampling rate of $173.61 \mathrm{~Hz}$ using a 12-bit analog-to-digital converter and was band-pass filtered between 0.5 and $85 \mathrm{~Hz}$ at $12 \mathrm{~dB}$ per octave. Apart from the approval by the local medical ethics committee, informed consent for retrospective analysis of the EEG recordings was obtained from all patients.

Any EEG recording within a time window of $1 \mathrm{~h}$ before a seizure until $2 \mathrm{~h}$ after a seizure as well as periods containing artifacts were discarded. During the recordings, patients were awake and at rest. No further preselection of the recordings was carried out. In total, we analyzed 84 EEG recordings [19]. Across patients, the total recording duration ranged from 19 to $585 \mathrm{~min}$, with an average of $130 \mathrm{~min}$ per patient. All recordings were performed prior to, and independent of, the design of this study and our previous study [19]. For further clinical details regarding the patient group and EEG recordings, see [19].

Since all patients were seizure free after unilateral resection of the hippocampal formation, the focal and nonfocal hemispheres are known for each patient and can be used as a gold standard to validate the performance of each measure under controlled conditions.

\section{Moving window analysis and patient-wise evaluation of results}

The steps of analysis described in this section were carried out for each individual patient and for all three measures separately. We use the symbol $M$ as a placeholder for the measures $C, L$, and $K$. We performed the analysis using a moving window technique with nonoverlapping windows of 4096 samples, corresponding to 23.6 s. For each window with time index $w$, we carried out the following steps of analysis. For each of the two electrodes, we calculated the measures $M$ for the 45 pairings of time series recorded with the 10 contacts. Pairings of contacts across the two hemispheres were not considered. We averaged results separately for the electrodes in the focal and nonfocal hemispheres. These averages are denoted by $M_{w}^{F}$ and $M_{w}^{N}$, respectively. For a few datasets, single contacts of an electrode were broken and therefore missing in the recordings. Note that values of $M$ can depend systematically on the distance between contacts along the axis of the electrodes. Accordingly, in order to have an unbiased comparison between the averages of the focal and nonfocal hemispheres, in cases of a broken contact, we also removed data from the homologous contact of the electrode in the opposite brain hemisphere from the analysis and took the averages of $M_{w}^{F}$ and $M_{w}^{N}$ only across the remaining symmetric subsets of contacts. For each window, we determined

$$
\Delta M_{w}=\frac{M_{w}^{F}-M_{w}^{N}}{M_{w}^{F}+M_{w}^{N}} .
$$

We use this normalized difference to facilitate the comparison across different measures. [The normalization of this expression relies on $M_{w}^{F, N}>0$. Even though $C$ and $K$ can attain zero values and $L$ can attain negative values, in practice, exclusively positive mean values $M_{w}^{F, N}>0$ were obtained. Hence, Eq. (8) can be applied.] Evidently, we get $\Delta M_{w} \rightarrow-1,0,1$ for $M_{w}^{F} \ll M_{w}^{N}, M_{w}^{F} \approx M_{w}^{N}$, and $M_{w}^{F} \gg M_{w}^{N}$, respectively. We use angular brackets to denote the average (we obtained very similar results when we at first took the average across windows and only then the normalized difference between the focal and nonfocal hemispheres) of $\Delta M_{w}$ across all windows: $\langle\Delta M\rangle$.

Suppose a certain measure $M$ was completely unspecific with regard to the dynamics of the focal versus the nonfocal hemisphere. In this case, $M_{w}^{F}$ and $M_{w}^{N}$ would follow the same distribution, and the mean value $\langle\Delta M\rangle$ would tend to zero for a large number of windows. However, for any finite number of windows, typically some nonzero $\langle\Delta M\rangle$ will be obtained. The expected magnitude of this $\langle\Delta M\rangle$ would not only depend on the total number of windows, but also in some nontrivial way on the particular shape of the distributions $M_{w}^{F, N}$ across windows and on the degree of correlations between results for subsequent analysis windows. Hence, a general analytical significance threshold for $\langle\Delta M\rangle$ can not be given.

As an alternative, one could consider to use a Monte Carlo resampling procedure to numerically derive a significance threshold for $\langle\Delta M\rangle$. However, it is not straightforward to account for the correlations between results for subsequent analysis windows in such a resampling procedure, and the degree of these correlations is different for the different measures $C, L$, and $K$. [We calculated the linear Pearson correlation coefficient between $\Delta M_{w}$ and $\Delta M_{w+1}$ across windows $w$ for all $84 \mathrm{EEG}$ recordings. Across these recordings, we found the following mean correlation coefficients $(\langle c\rangle)$ and numbers of significant correlation coefficients $\left(\# p_{c}<0.05\right)$. Linear cross correlation $C:\langle c\rangle=0.29, \# p_{c}<0.05=51$ of 84; nonlinear interdependence measure $\mathrm{L}:\langle c\rangle=0.24$, $\# p_{c}<0.05=42$ of 84 ; surrogate-corrected nonlinear interdependence measure $\mathrm{K}:\langle c\rangle=0.15, \# p_{c}<0.05=28$ of 84 .] We therefore restrict ourselves to the statistics described in the following section to assess the significance of the results across patients. 


\section{Patient group statistics}

Here we denote by $\langle\Delta M\rangle^{(v)}$ the average across windows for an individual patient with index $v$. In contrast to the $\Delta M_{w}$ across windows with index $w$, the $\langle\Delta M\rangle^{(v)}$ across patients are independent samples. The mean value across all 29 patients is denoted by $\overline{\langle\Delta M\rangle}$. We test here the null hypothesis that $M$ is completely unspecific with regard to the focal and nonfocal hemispheres in two different ways. Given a certain number of positive $\langle\Delta M\rangle^{(v)}$ values, we use the binomial distribution to determine the probability $p_{M}^{\text {sign }}$ to obtain this or a higher number of positive values in 29 independent trials with a hit probability of $50 \%$. This probability $p_{M}^{\text {sign }}$ only takes the sign of $\langle\Delta M\rangle^{(v)}$ into account. To also evaluate the magnitude of these $\langle\Delta M\rangle^{(v)}$ values, we used a simple Monte Carlo simulation based on a random switching of results for the focal and nonfocal hemispheres:

$$
{\overline{\langle\Delta M\rangle_{\text {rand }}}}=\frac{1}{29} \sum_{v=1}^{29} r_{v}\langle\Delta M\rangle^{(v)}
$$

with

$$
r_{v}=\left\{\begin{array}{cc}
1 & \text { with probability } 0.5 \\
-1 & \text { else }
\end{array}\right.
$$

We calculated an arbitrary but high number of $10^{9}-1$ independent realizations of $\overline{\langle\Delta M\rangle}$ rand and determined the quantile that $\overline{\langle\Delta M\rangle}$ fell within the resulting distribution across realizations. If the null hypothesis of the measures' unspecificness was true, the probability $p_{M}^{\mathrm{val}}$ to get $\overline{\langle\Delta M\rangle}$ or a higher result is given by the complementary of this quantile.

\section{E. Choice of parameters}

We used $\kappa=5$ nearest neighbors and a Theiler window of $W=25$. No other values for $\kappa$ or $W$ were tested. To set the values of the embedding dimension $m$ and time delay $\tau_{D}$, we started out by analyzing individual windows of EEG time series and single data sets from patients 3 and 29. For this preanalysis, we scanned the ranges $m=$ $[2,3,4, \ldots, 20] \times \tau_{D}=[2,4,6, \ldots, 20]$. We found stable results of $\Delta L_{w}, \Delta K_{w},\langle\Delta L\rangle^{(v)}$, and $\langle\Delta K\rangle^{(v)}$ for the approximate range $m=[8, \ldots, 12] \times \tau_{D}=[10,12,14]$. In contrast, for higher $\left(m \rightarrow 20, \tau_{D} \rightarrow 20\right)$ and lower $\left(m \rightarrow 2, \tau_{D} \rightarrow 2\right)$ values, small changes in either $m$ or $\tau_{D}$ resulted in more substantial changes of $\Delta L_{w}, \Delta K_{w},\langle\Delta L\rangle^{(v)}$, and $\langle\Delta K\rangle^{(v)}$. We therefore set the parameters to the middle of the stable regime, i.e., $m=10$ and $\tau_{D}=12$, and provide detailed results for these values in Sec. III. To further test the robustness of our results against changes of the embedding parameters, we calculated the results across patients $\left(\overline{\langle\Delta L\rangle}, p_{L}^{\text {sign }}, p_{L}^{\text {val }}, \overline{\langle\Delta K\rangle}, p_{K}^{\text {sign }}\right.$, and $\left.p_{K}^{\mathrm{val}}\right)$ for ranges of $m=[8, \ldots, 12] \times \tau_{D}=[10,12,14]$ and exemplary settings of very low $\left(m=2, \tau_{D}=2\right)$ and very high ( $m=20, \tau_{D}=20$ ) values of the embedding parameters. Consistently with the results of the preanalysis, we found stable results within the range $m=[9, \ldots, 12] \times \tau_{D}=[10,12,14]$ (see Table I in Appendix B). In contrast, deviations from the results obtained for $m=10$ and $\tau_{D}=12$ were found for $m=8 \times \tau_{D}=[10,12,14]$ and, in particular, for the exemplary settings of very low and very high values of the embedding parameters. Importantly, while testing the robustness of our results against changes in the parameters, we did not carry out any kind of parameter optimization with regard to the results presented below. In fact, maximal values for $\overline{\langle\Delta L\rangle}$ and $\overline{\langle\Delta K\rangle}$ as well as minimal values for $p_{L}^{\text {sign }}, p_{L}^{\mathrm{val}}, p_{K}^{\text {sign }}$, and $p_{K}^{\mathrm{val}}$ were found for parameter values different from $m=10$ and $\tau_{D}=12$ (see Appendix B).

\section{RESULTS}

Exemplary segments of EEG recordings along with the values of $C, L$, and $K$ obtained for these segments are shown in Figs. 2-4. Common to these examples is a prominent similarity between the patterns in the matrices of $C$ and $L$. This similarity reflects the well-known strong impact of linear cross correlation on nonlinear interdependence measures [18]. In contrast, there is only little resemblance between the matrices of $K$ versus those of $C$ and $L$. We furthermore note a segmentation of the matrices of $C$ and $L$. Prominent square structures next to the diagonal reflect that, for these two measures in general, higher dependencies are found within certain channel groups as opposed to channel combinations across these groups. This segmentation reflects that the different channel groups are located in different anatomical substructures of the hippocampal formation and that EEG recordings from within the same substructure are mutually correlated (cf. [6,33]). In general, no such clear segmentation is found in the matrices of $K$.

As described in the Introduction, it can be conjectured that the epileptic process is related to an elevated level of neuronal synchronization that persists in the absence of actual seizure activity. Accordingly, the interdependence between recordings from within the focal hemisphere is expected to be higher than that between recordings from within the nonfocal hemisphere. We therefore expect to obtain positive values for $\Delta M_{w}$ and the derived mean values $\langle\Delta M\rangle^{(v)}$ and $\overline{\langle\Delta M\rangle}$. Values of $\langle\Delta C\rangle^{(v)}$ are positive for 19 of the 29 patients (Fig. 5). However, the probability to have 19 or more positive values by chance amounts to $p_{C}^{\text {sign }}=0.068$. Hence, regarding the fraction of positive values, results for the linear cross correlation $C$ are not significant at a level of $\alpha=0.05$. The strong impact of linear cross correlation on nonlinear interdependence measures is further evidenced by a very strong correlation between $\langle\Delta C\rangle^{(v)}$ and $\langle\Delta L\rangle^{(v)}$ across patients (Fig. 5). Nonetheless, results for the nonlinear interdependence measure $L$ turn out to be significant. We observe 23 positive $\langle\Delta L\rangle^{(v)}$ values $\left(p_{L}^{\text {sign }}=0.0012\right)$. A far higher contrast between the focal and nonfocal hemispheres is obtained once we apply the surrogate correction to the nonlinear interdependence measure. We observe positive $\langle\Delta K\rangle^{(v)}$ values for 27 out of 29 patients $\left(p_{K}^{\text {sign }}=8.1 \times 10^{-7}\right)$.

The overall magnitude of $\langle\Delta K\rangle^{(v)}$ values is higher than that of $\langle\Delta C\rangle^{(v)}$ and $\langle\Delta L\rangle^{(v)}$ (Figs. 5 and 6). For the linear cross correlation, the average across patients results in $\overline{\langle\Delta C\rangle}=0.026$. This, or a higher mean value, is expected with probability $p_{C}^{\mathrm{val}}=0.078$, given the distribution of the 29 values of $\langle\Delta C\rangle^{(v)}$ (Fig. 6). Hence, also when averaged across patients, the results for the linear cross correlation 


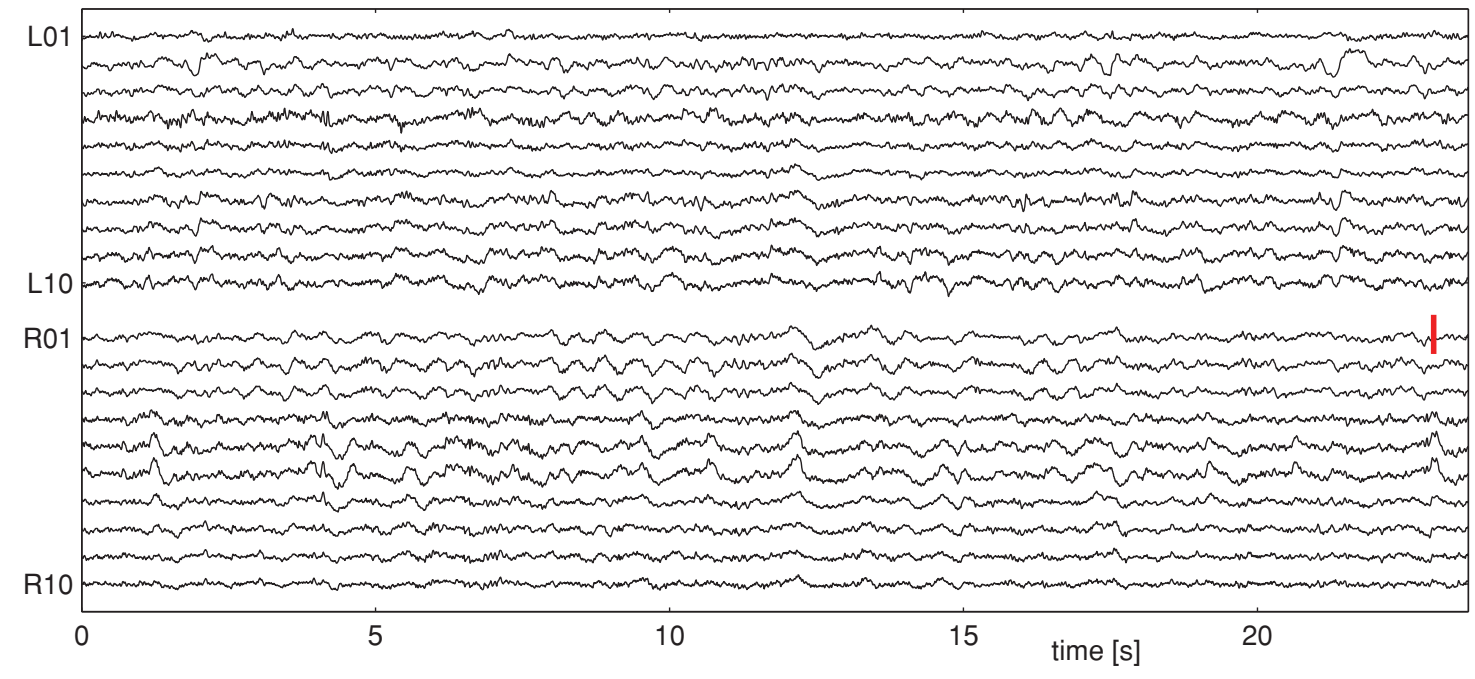

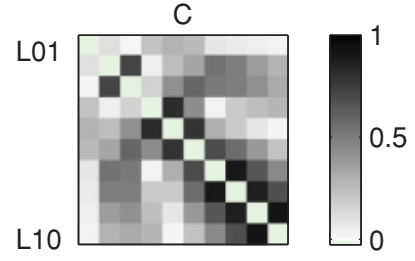

L01

L10

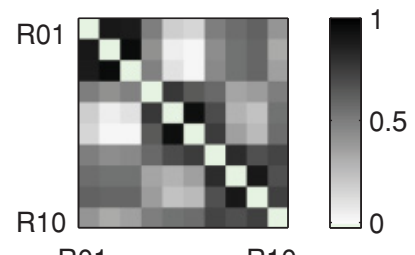

R01

R10

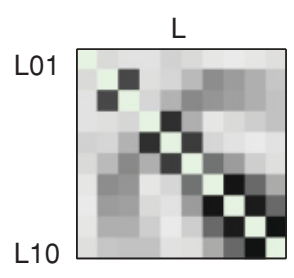

L01

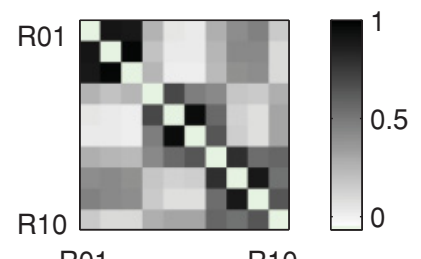

R01

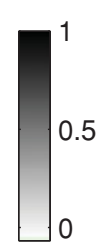

L10

R10
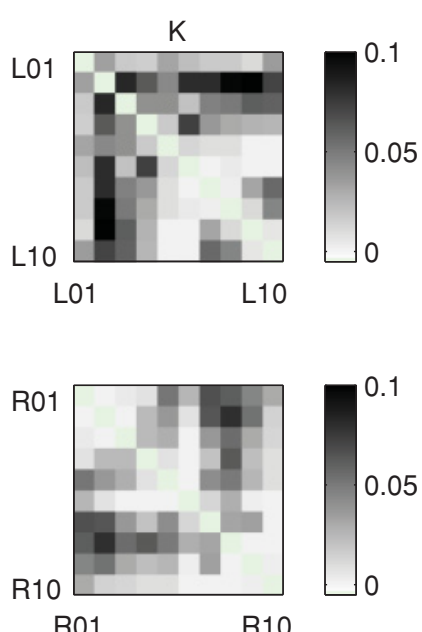

R01

FIG. 2. (Color online) Top: Exemplary window of an EEG recording. The scale bar on the right indicates $500 \mu \mathrm{V}$. For this particular patient, the epileptic focus was in the hippocampal formation of the left brain hemisphere, and we obtain $\langle\Delta C\rangle^{(v=16)}<0,\langle\Delta L\rangle^{(v=16)}<0$, but $\langle\Delta K\rangle^{(v=16)}>0$ (see Fig. 5). Bottom: Grayscaled values of the different measures obtained for this EEG window. By construction, all these result matrices are symmetric. The diagonals are left empty. See Fig. 1 for the channel labeling.

remain not significant at a level of 0.05 . In contrast, we again obtain significant results for the nonlinear interdependence measure: $\overline{\langle\Delta L\rangle}=0.074\left(p_{L}^{\mathrm{val}}=4.6 \times 10^{-4}\right)$. Again, the highest contrast between results for the focal and nonfocal hemispheres is obtained for the surrogate-corrected nonlinear interdependence measure, yielding $\overline{\langle\Delta K\rangle}=0.269$. This value of $\overline{\langle\Delta K\rangle}$ is located in the very tail of the distribution $\overline{\langle\Delta K\rangle}_{\text {rand }}$ (see Fig. 6). Under the null hypothesis that $K$ was unspecific with regard to focal and nonfocal hemispheres, we expect this, or a higher value, with a probability of only $p_{K}^{\mathrm{val}}=6.5 \times 10^{-7}$. We found no discernible dependence of $\langle\Delta C\rangle^{(v)},\langle\Delta L\rangle^{(v)}$, or $\langle\Delta K\rangle^{(v)}$ on the total recording duration across patients. Testing for linear dependencies between these values and the total recording duration, we obtained only low and nonsignificant values of Pearson correlation coefficients $\left(\langle\Delta C\rangle^{(v)}: c=0.05, p_{c}=\right.$ $0.78 ;\langle\Delta L\rangle^{(v)}: c=-0.10, p_{c}=0.62 ;\langle\Delta K\rangle^{(v)}: c=-0.25$, $\left.p_{c}=0.20\right)$.

\section{DISCUSSION}

We summarize that all three bivariate interdependence measures tested here showed higher average values for EEG recordings from the focal brain hemisphere than for those from the nonfocal hemisphere. This general result is congruent with those reported in earlier studies [1-9]. For the linear cross correlation $C$, however, the differences between the focal and nonfocal hemispheres were found to be not significant. Results for the nonlinear interdependence measure $L$ were significant, but still had only a moderate accuracy in determining the side of the focal hemisphere. The highest accuracy and significance was obtained for the surrogate-corrected nonlinear interdependence measure $K$. Hence, a specific result of our study is that the bivariate surrogate correction seems key to a successful characterization of the spatial distribution of the epileptic process.

We emphasize that this surrogate correction is conceptually very straightforward. One calculates the nonlinear 


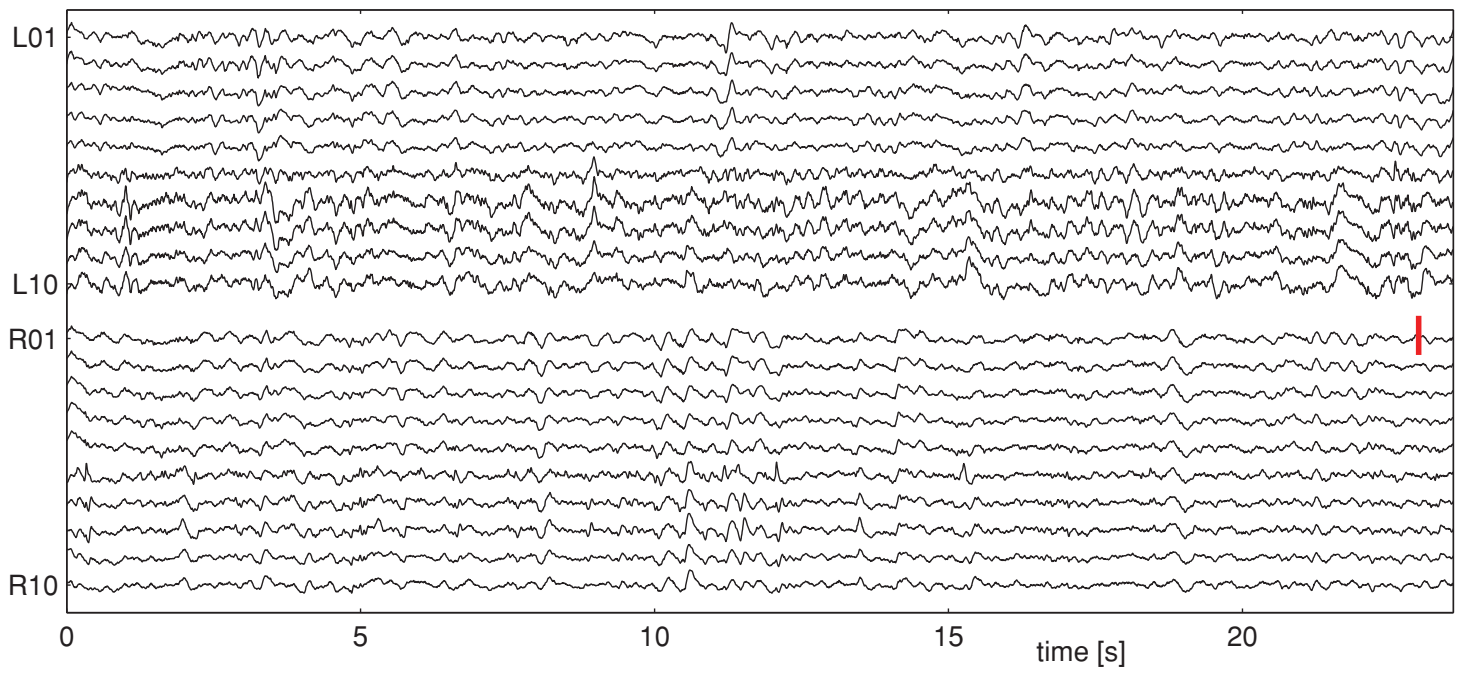

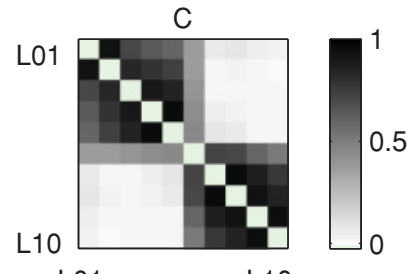

L01

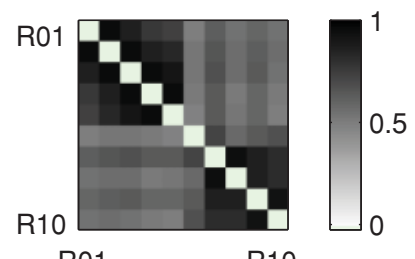

$\mathrm{R} 01$

R10

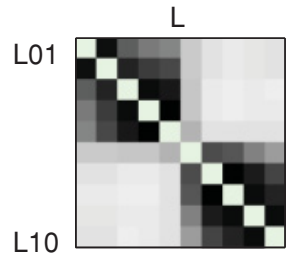

L01

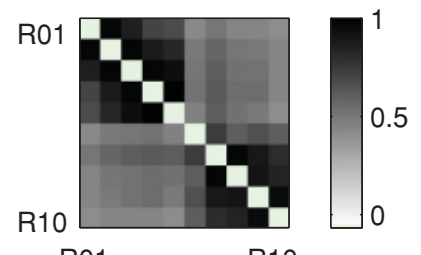

$\mathrm{R} 01$

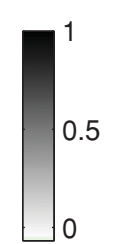

L10

R10
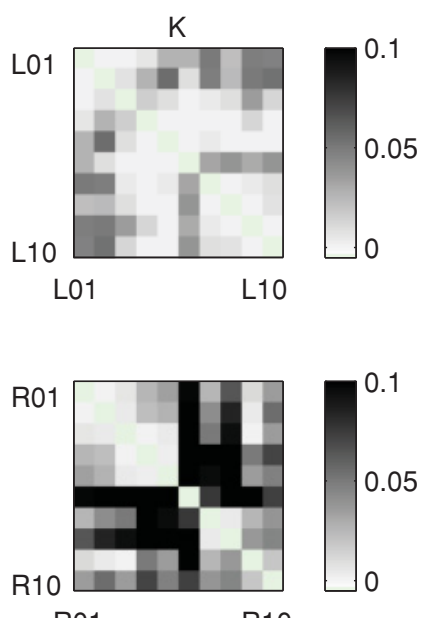

R01

R10

FIG. 3. (Color online) EEG window from another patient and corresponding results. Same organization as in Fig. 2. For this patient, the epileptic focus was in the hippocampal formation of the right brain hemisphere, and we obtain $\langle\Delta C\rangle^{(v=22)}>0,\langle\Delta L\rangle^{(v=22)}>0$, and $\langle\Delta K\rangle^{(v=22)}>0$ (see Fig. 5).

interdependence measure not only for the original EEG time series, but also for an ensemble of surrogate time series. The surrogate-corrected nonlinear measure is defined by the rectified difference between the results for the original time series and the mean result for the surrogates [Eq. (7)]. The exact definition of this difference is not crucial. We obtained very similar results when the maximum rather than the mean of the surrogates was used or when the $K$ values were not rectified to non-negative values. Importantly, a surrogate correction can be applied to any nonlinear or linear [39] time series analysis measure.

The bivariate surrogates used here represent the null hypothesis $\mathcal{H}_{0 \text {,biv }}: X$ and $Y$ jointly represent a stationary bivariate linear stochastic correlated Gaussian process. The measurement functions by which $x_{n}$ and $y_{n}$ were derived from the dynamics are invertible but potentially nonlinear. The autocorrelation, cross correlation, mean, and variance of the underlying Gaussian process are such that the measurement results in the autocorrelation, cross correlations, and amplitude distribution of the observed time series. A main result of our study is that the EEG recorded in the focal hemisphere is less consistent with this null hypothesis than the EEG recorded in the nonfocal hemisphere. As a possible conclusion, let us consider the following hypothesis $\mathcal{H}_{1 \text {,biv }}$ : The dynamics of neuronal groups in the focal and nonfocal hemispheres have nonlinear deterministic properties, and the coupling between neuronal groups is on average stronger in the focal hemisphere than in the nonfocal hemisphere. Does the rejection of $\mathcal{H}_{0 \text {,biv }}$ prove the correctness of $\mathcal{H}_{1 \text {,biv }}$ ? The answer is no. The null hypothesis $\mathcal{H}_{0 \text {,biv }}$ is composed of a number of distinct assumptions, and the violation of any of these assumptions is by itself sufficient for a rejection of $\mathcal{H}_{0 \text {,biv }}$. Hence, one of many possible alternatives to $\mathcal{H}_{1 \text {,biv }}$ could be that the dynamics in the focal and nonfocal hemispheres are consistent with a bivariate linear stochastic process, but the dynamics of the focal hemisphere is more nonstationary. The assumption of stationarity is not only included in the surrogate null hypothesis, but it also underlies the calculation of the nonlinear interdependence measure $L$. A variety of physiological mechanisms can cause 


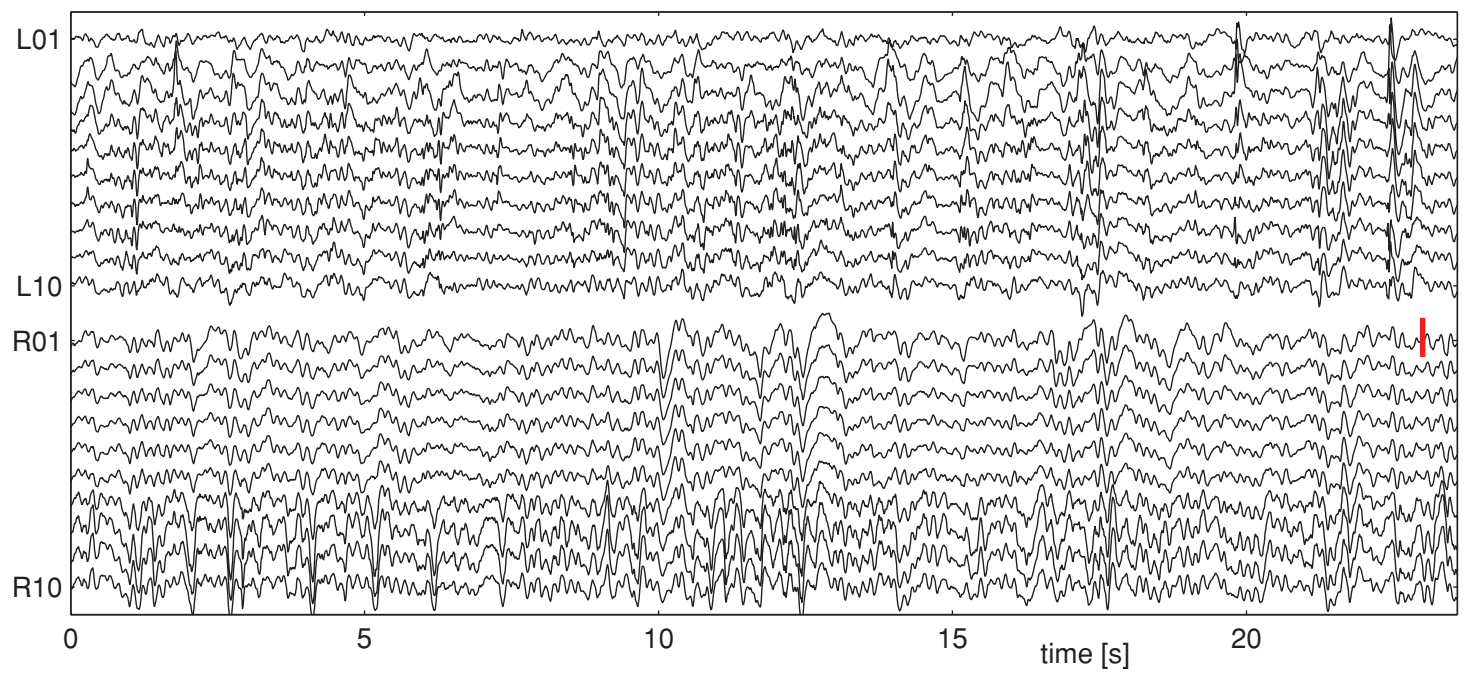

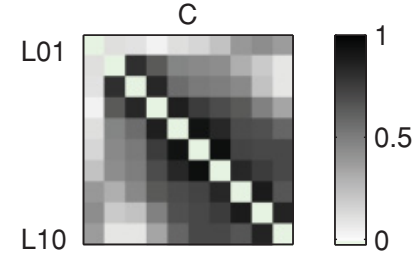

L01

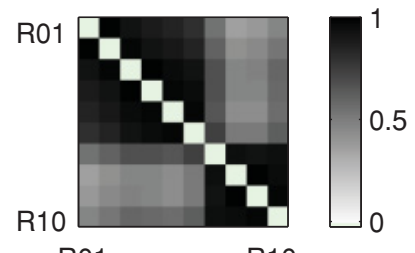

R01

R10

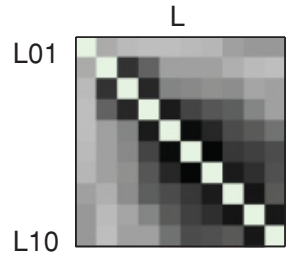

L01

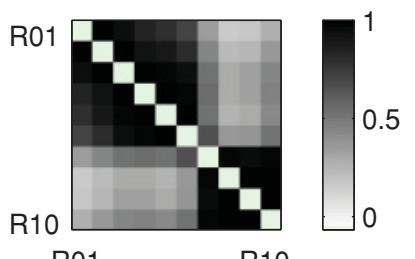

R01

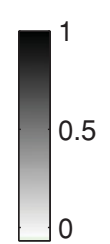

L10

R10
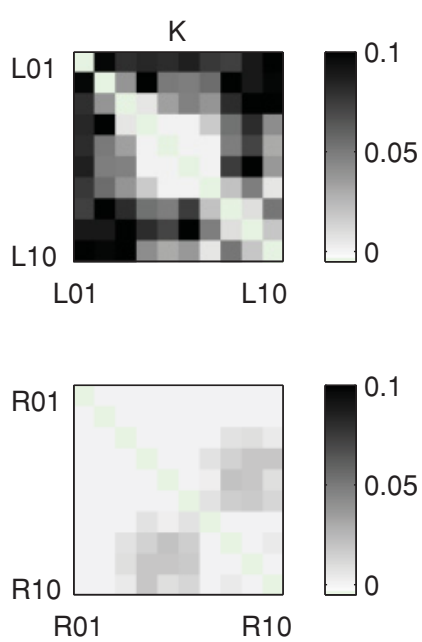

FIG. 4. (Color online) EEG window from another patient and corresponding results. Same layout as in Figs. 2 and 3 . For this patient, the epileptic focus was in the hippocampal formation of the right brain hemisphere, and we obtain $\langle\Delta C\rangle^{(v=27)}>0,\langle\Delta L\rangle^{(v=27)}>0$, but $\langle\Delta K\rangle^{(v=27)}<0$ (see Fig. 5).

nonstationarities that are reflected in the EEG on a range of different time scales. Intermittent interictal epileptiform activity during the seizure-free interval and the reduction of antiepileptic medication during the presurgical epilepsy diagnostics (cf. [34]) can lead to further nonstationary features that are evident on different time scales. In consequence, regardless of the window length used for the analysis, the assumption of stationarity can not be fulfilled in a strict sense. Furthermore, we have to keep in mind that the EEG results from the superposition of electrical potentials of a huge number of individual neurons. These neurons interact in local circuits as well as with remote neurons. Hence, EEG time series recorded at individual contacts incorporate a large number of degrees of freedom. Accordingly, delay coordinates with some limited embedding dimension will probably not suffice to obtain a reasonably projection-free reconstruction of the underlying dynamics. The embedding dimension can not, on the other hand, be increased arbitrarily due to the limited length of the time series. Indeed, the highest accuracy and significance of the measure $K$ in determining the side of the focal hemisphere was found for intermediate values of the embedding dimension, while both low and high values of this parameter led to a weaker performance.

Seeking a further interpretation of our results, we turn to previous findings of ours [19] (see also [20,21]). Based on the same set of EEG recordings studied here, this previous study determined the degree to which different univariate time series analysis approaches allowed us to determine the side of the focal hemisphere. We found that the accuracy of both linear and nonlinear univariate measures was low if not insignificant. In contrast, a high accuracy was obtained for univariate surrogate-corrected nonlinear measures. In particular, the focal EEG was less consistent with the surrogates' null hypothesis than the nonfocal EEG. Accordingly, our previous study [19] and this study have an important conclusion in common. A combination of nonlinear measures with surrogates allows one to extract information from experimental time series that can not be extracted using the nonlinear measures alone. This is remark- 


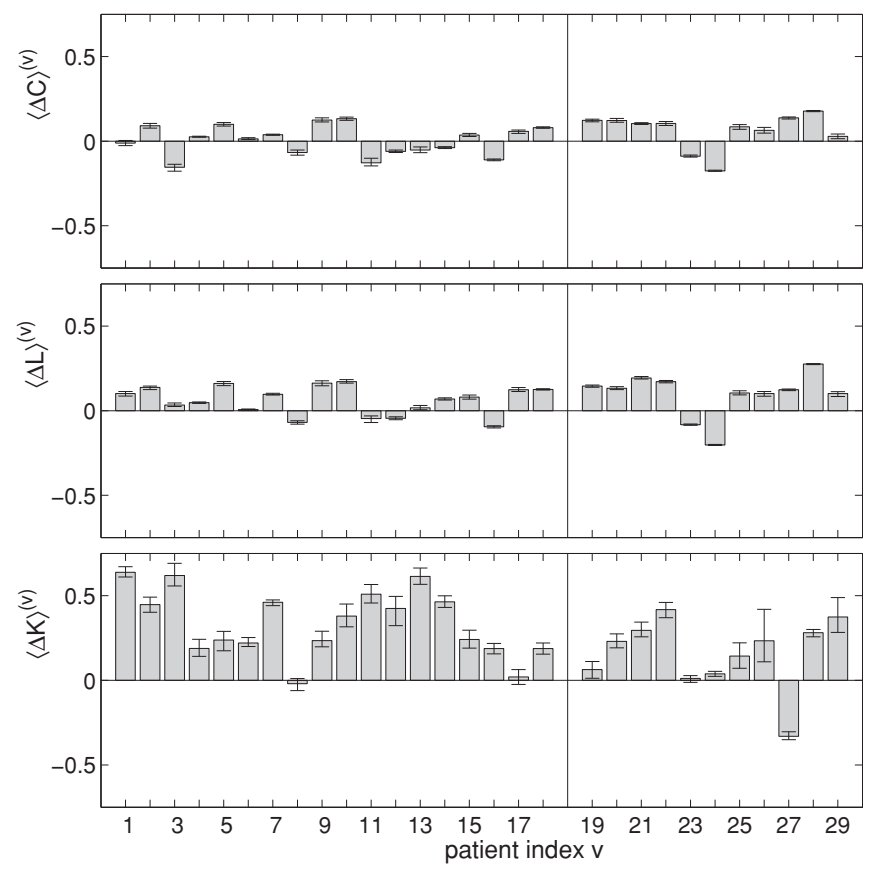

FIG. 5. Results for individual patients. Values of $\langle\Delta M\rangle^{(v)}$ for patients with the focus in the left hemisphere (patients $v=1, \ldots, 18$ ) and right hemisphere (patients $v=19, \ldots, 29$ ). For reasons given in Sec. II C, it is not possible to provide reliable confidence levels for these mean values. To still provide an estimate of the certainty of these values, we composed subsamples by randomly picking $66 \%$ of all available windows for individual patients and determined the $\langle\Delta M\rangle^{(v)}$ values for these random subsamples. The error bars indicate ranges of $\langle\Delta M\rangle^{(v)}$ obtained for 100 independent realizations of the random subsamples. The patient index corresponds to the one used in Fig. 4 of Ref. [19].
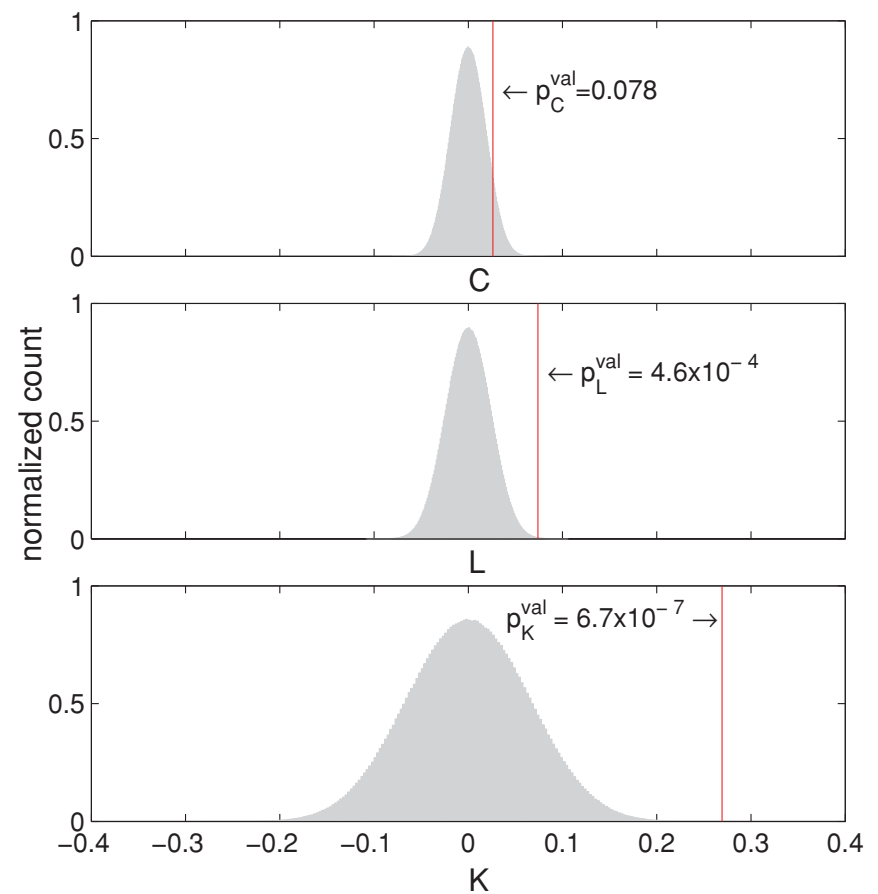

FIG. 6. (Color online) Results averaged across patients: Values of $\overline{\langle\Delta M\rangle}$ are shown as vertical lines. Distributions of $\overline{\langle\Delta M\rangle}_{\text {rand }}$ across $10^{9}-1$ independent realizations of Eq. (9) are shown in gray. able since univariate and bivariate time series analysis are based on conceptually different approaches. While univariate analysis characterizes local aspects of the dynamics, bivariate analysis characterizes the interaction between different dynamics.

The null hypothesis tested by the particular univariate surrogates we used in [19] is that the dynamics are a univariate linear stationary Gaussian stochastic process and that the time series were recorded using an invertible but potentially nonlinear measurement function. The autocorrelation, mean, and variance of the underlying Gaussian process are such that the measurement results in the autocorrelation and amplitude distribution of the observed time series $\left(\mathcal{H}_{0 \text {, univ }}\right)$ [35]. Hence, these surrogates are the univariate analog to the bivariate surrogates used here. The difference is that the bivariate surrogates account for possible cross correlations between $X$ and $Y$. Nonetheless, a violation of the univariate null hypothesis for either $X$ or $Y$ implies a violation of the bivariate null hypothesis for the pair $X$ and $Y$. This holds independent of the degree of dependence between the two dynamics. If, for example, $X$ and $Y$ are independent, but one or both of them exhibit nonlinear deterministic structures, then both the univariate and the bivariate null hypotheses are violated. Hence, taking [19] into account, a possible explanation of our findings obtained from bivariate measures is that the focal EEG is less consistent with a linear stationary Gaussian stochastic process than the nonfocal EEG. These considerations could imply that the results of our bivariate analysis mainly reflect properties of the individual univariate EEG time series rather than any signatures of coupling or nonlinear interdependence between pairs of time series. Indeed, there is a noticeable correlation between the results of $K$ and those of univariate surrogate-corrected measures (compare Fig. 5 with Fig. 3 in Ref. [19]). [To appreciate this, one has to calculate the normalized difference of the univariate surrogate-corrected measure values shown in Fig. 3 of Ref. [19] according to Eq. (8). The Pearson correlation coefficient between the resulting values and the $\langle\Delta K\rangle^{(v)}$ values across patients is $c=0.61\left(p_{c}=\right.$ 0.0004).]

We, therefore, studied the potential influence of properties of the individual univariate EEG time series on both $L$ and $K$ by using time-shifted surrogates [18,24,36]. Similar to so-called twin surrogates [37], time-shifted surrogates represent the null hypothesis $\mathcal{H}_{0 \text {,biv }}^{*}$ that the two dynamics are independent and not cross correlated. No assumption is made about the structure of the individual time series. We constructed individual pairs of time-shifted surrogates by performing a cycle shift of the $y_{n}$ time series by a random interval of at least $1 \mathrm{~s}$ relative to the $x_{n}$ time series. The mean $L$ value obtained from 19 pairs of time-shifted surrogates, denoted by $\left\langle L_{S}^{*}\right\rangle$, was used to replace $\left\langle L_{S}\right\rangle$ in Eq. (7) resulting in $K^{*}$. All subsequent steps of analysis were kept the same. The $\left\langle\Delta K^{*}\right\rangle^{v}$ values were found to be almost identical to those of $\langle\Delta L\rangle^{v}$. The Pearson correlation coefficient between these sets of values across patients was $c=0.9991$. (While this close match of $\left\langle\Delta K^{*}\right\rangle^{v}$ and $\langle\Delta L\rangle^{v}$ has strong implications for the interpretation of our main findings, it also implies that a description of the detailed results obtained from time-shifted surrogates would not provide 
additional information. For the sake of better readability and brevity, we therefore limited the description of the study on time-shifted surrogates to this short exposition embedded in the discussion of our main findings.) The reason is that $K^{*}$ was, in general, close to $L_{O}$ since $\left\langle L_{S}^{*}\right\rangle$ was, in general, close to zero, which means that a surrogate correction for $L$ based on time-shifted surrogates has basically no effect. This is to be expected since time-shifted surrogates represent the null hypothesis of independent dynamics, and the measure $L$ is defined to have an expected value of zero for such independent dynamics. It has already been shown for exemplary mathematical model systems that $L$ is less sensitive to properties of the individual time series and more specific for signatures of nonlinear interdependencies than other bivariate nonlinear interdependence measures [28]. The fact that $\left\langle L_{S}^{*}\right\rangle$ was, in general, close to zero shows that, also for the specific case of the EEG recordings studied here, $L$ is not sensitive to properties of the individual univariate time series. Accordingly, while a violation of the univariate null hypothesis $\mathcal{H}_{0 \text {, univ }}$ for either $X$ or $Y$ indeed formally implies a violation of the bivariate null hypothesis $\mathcal{H}_{0 \text {, biv }}$ for the pair $X$ and $Y$, this violation is not likely to result in a nonzero value of $K$.

In consequence, results from the univariate analysis presented in [19] and the bivariate analysis presented here provide complementary perspectives on the dynamics of the epileptic process. As outlined above, the epileptic process is generally considered to induce hypersynchronous activity of neuronal groups. This hypersynchronicity can have different manifestations on different spatial scales of neuronal organization. For univariate signals recorded by individual contacts, it can result in a more regular appearance of the EEG. For bivariate signals recorded by pairs of contacts, it can result in stronger signatures of interdependence. Across these spatial scales, the hypersynchronicity assumed to be induced by the epileptic process can result in an EEG that is less consistent with a linear stochastic process and, rather, exhibits some properties of a coupled nonlinear deterministic dynamics.

We recall that, for our group of patients with unilateral medial temporal lobe epilepsy, the clinical presurgical diagnostics allowed us to determine whether the left or right hippocampal formation contained the epileptic focus. The EEG was recorded with one depth electrode per hippocampal formation (Fig. 1). The resection of the focal hippocampal formation led to complete seizure control in all patients. Accordingly, we compared averaged results at the level of the two depth electrodes in order to retrospectively determine the side of the focal brain hemisphere. The high accuracy of the surrogate-corrected nonlinear interdependence measure $K$ shows that this approach carries the potential to extract valuable information from the EEG of epilepsy patients. A thorough assessment of the potential of such an analysis to contribute to the presurgical diagnosis of epilepsy patients certainly requires further comprehensive studies. First of all, beyond a determination of the side of the focal hemisphere, a precise localization of the epileptic focus down to the spatial resolution of individual recording contacts should be aimed for. This task can be addressed, for example, by studying patients with neocortical epilepsies who are implanted with laminar subdural grid electrodes. These electrodes are equipped with grids of, e.g., $8 \times 8$, recording contacts and are implanted beneath the dura mater onto the surface of the cortex. Typically, brain areas covered by only a small number of individual contacts are identified as the epileptic focus and resected. Hence, these cases can be used to study the capability of surrogate-corrected nonlinear interdependence measures for a precise localization of epileptic foci.

Beyond different types of epilepsies, future work should study the impact of various factors on the discriminative power of the different time series analysis techniques in localizing the epileptic focus. These influencing factors can be, for example, different states of vigilance or levels of anticonvulsive medication. Aspects of the current clinical status and medical history of individual patients, such as the mean frequency of seizures or duration of the disease, are of interest. Future work should also include patients for whom epilepsy surgery did not lead to complete seizure control as negative controls. An important methodological aspect of such future work will be to evaluate not only the strength, but also the direction of interactions between different brain areas [10-13]. This work should also include advanced measures based on Granger causality (see [38] and references therein). Leading beyond the spatial averages studied here, such analysis can yield weighted and directed interaction networks (for an overview, see [38,39] and references therein). This approach can allow for an advanced characterization of the spatiotemporal dynamics of the epileptic focus and its interactions with other brain areas.

\section{ACKNOWLEDGMENTS}

R.G.A acknowledges support from Grant No. BFU200761710 of the Spanish Ministry of Education and Science. D.C. was supported by the Grant No. 2008FI-B 00460 of the Generalitat de Catalunya and European Social Funds. K.L. acknowledges support from the Deutsche Forschungsgemeinschaft (Grant No. LE660/5-1). F.M. acknowledges support from the European Commission (Marie Currie OIF 040445) and the Jung Foundation for Science and Research.

\section{APPENDIX A}

We used the following iteration scheme [17] to generate bivariate surrogates. Let $a_{k, x} e^{i \phi_{k, x}}$ and $a_{k, y} e^{i \phi_{k, y}}$ denote the discrete Fourier transforms of $x_{n}$ and $y_{n}(k=-N / 2, \ldots, N / 2$, and $n=0, \ldots, N-1$; for simplicity, we assume $N$ to be even). To generate a seed for the iteration scheme, the temporal order of the time series $x_{n}$ and $y_{n}$ is permuted, resulting in $\widetilde{x}_{n}$ and $\tilde{y}_{n}$. Importantly, two different unconstrained permutations are used for $X$ and $Y$. Each iteration step consists of the following two procedures:

(i) Filtering. Calculate the discrete Fourier transform $b_{k, x} e^{i \psi_{k, x}}$ of $\widetilde{x}_{n}$ and replace the amplitudes with the original amplitudes $a_{k, x}$. The random phases $\psi_{k, x}$ are replaced with new phases $\widetilde{\psi}_{k, x}$, which are specified below. Take the inverse transform of $a_{k, x} e^{i \tilde{\psi}_{k, x}}$ resulting in $\tilde{x}_{n}^{*}$. Carry out the same steps for $Y$. The use of the original amplitudes $a_{k, x}$ and 
$a_{k, y}$ imposes the periodograms of the original time series to $\tilde{x}_{n}^{*}$ and $\tilde{y}_{n}^{*}$. The phases of the individual surrogates, on the other hand, should remain random. Therefore, one aims to minimize

$$
h_{k}=\left|e^{i \tilde{\psi}_{k, x}}-e^{i \psi_{k, x}}\right|^{2}+\left|e^{i \tilde{\psi}_{k, y}}-e^{i \psi_{k, y}}\right|^{2} .
$$

At the same time, to preserve the cross correlation, the circular differences between the surrogate phases must coincide with the original ones [40]:

$$
e^{i\left(\widetilde{\psi}_{k, x}-\widetilde{\psi}_{k, y}\right)}=e^{i\left(\phi_{k, x}-\phi_{k, y}\right)},
$$

which is fulfilled if we choose $\widetilde{\psi}_{k, x}$ and $\widetilde{\psi}_{k, y}$ such that

$$
\widetilde{\psi}_{k, x}-\phi_{k, x}=\widetilde{\psi}_{k, y}-\phi_{k, y}=\alpha_{k}
$$

A value for $\alpha_{k}$ is found by first eliminating $\widetilde{\psi}_{k, x}$ and $\widetilde{\psi}_{k, y}$ from Eq. (A1), which turns into

$$
h_{k}=4-2 \cos \left(\alpha_{k}-\psi_{k, x}+\phi_{k, x}\right)-2 \cos \left(\alpha_{k}-\psi_{k, y}+\phi_{k, y}\right) \text {. }
$$

This $h_{k}$ is extremal for

$$
\tan \alpha_{k}=\frac{\sin \left(\psi_{k, x}-\phi_{k, x}\right)+\sin \left(\psi_{k, y}-\phi_{k, y}\right)}{\cos \left(\psi_{k, x}-\phi_{k, x}\right)+\cos \left(\psi_{k, y}-\phi_{k, y}\right)} .
$$

The solutions of Eq. (A5), which are $\left\{\alpha_{k}, \alpha_{k}+\pi, \alpha_{k}+\right.$ $2 \pi, \ldots\}$, correspond to alternating maxima and minima of
Eq. (A4), as can be seen from the second derivative of Eq. (A4). Hence, if the arctan function taken from Eq. (A5) results in a maximum of $h_{k}$, one has to add $\pi$ before inserting it in Eq. (A3) to determine $\widetilde{\psi}_{k, x}$ and $\widetilde{\psi}_{k, y}$. This is carried out for $0>k>N / 2$. The real-valued Fourier coefficients for $k=0$ and $k= \pm N / 2$ are not filtered. Phases for negative $k$ are set to $\widetilde{\psi}_{-k, x}=-\widetilde{\psi}_{k, x}$ and $\widetilde{\psi}_{-k, y}=-\widetilde{\psi}_{k, y}$ to preserve the symmetry of the discrete Fourier transform of real-valued time series. As a result of the filtering, the autocorrelation function and cross-correlation function of $\tilde{x}_{n}^{*}$ and $\tilde{y}_{n}^{*}$ coincide with those of the original time series. However, the amplitude values of $\tilde{x}_{n}^{*}$ and $\tilde{y}_{n}^{*}$ will no longer match the original ones.

(ii) Amplitude adjustment. To restore the original amplitude values in the time domain, replace the highest, second-highest, ..., smallest value of $\tilde{x}_{n}^{*}$ with the highest, second-highest, ..., smallest value value of $x_{n}$. Assign the result of this rescaling to $\tilde{x}_{n}$ and carry out the same steps for $Y$. After this adjustment, the amplitudes of $\tilde{x}_{n}$ and $\tilde{y}_{n}$ coincide with the original ones. However, the autocorrelation and cross-correlation functions are distorted. Therefore, the iteration scheme is continued with the filtering step using the reassigned $\tilde{x}_{n}$ and $\tilde{y}_{n}$ as input.

After a few dozen iterations, this scheme typically converges to diminishing distortions of the autocorrelation functions and the cross-correlation function after the amplitude adjustment step. We use here an arbitrary but high number of 120 iterations. By construction, the amplitudes of the

\begin{tabular}{|c|c|c|c|c|c|c|c|c|c|}
\hline$m$ & $\tau_{D}$ & $\langle\Delta L\rangle^{(v)}>0^{\mathrm{a}}$ & $p_{L}^{\text {sign }}$ & $\overline{\langle\Delta L\rangle}$ & $p_{L}^{\mathrm{val} \mathrm{b}}$ & $\langle\Delta K\rangle^{(v)}>0^{\mathrm{a}}$ & $p_{K}^{\text {sign }}$ & $\overline{\langle\Delta K\rangle}$ & $p_{K}^{\mathrm{val} \mathrm{b}}$ \\
\hline 2 & 2 & 22 & $4.1 \times 10^{-3}$ & 0.065 & $6.3 \times 10^{-3}$ & 24 & $2.7 \times 10^{-4}$ & 0.349 & $1.5 \times 10^{-6}$ \\
\hline 8 & 10 & 20 & $3.1 \times 10^{-2}$ & 0.068 & $1.4 \times 10^{-3}$ & 26 & $7.6 \times 10^{-6}$ & 0.220 & $8.2 \times 10^{-7}$ \\
\hline 8 & 12 & 23 & $1.2 \times 10^{-3}$ & 0.073 & $6.9 \times 10^{-4}$ & 26 & $7.6 \times 10^{-6}$ & 0.288 & $5.2 \times 10^{-7}$ \\
\hline 8 & 14 & 23 & $1.2 \times 10^{-3}$ & 0.070 & $1.1 \times 10^{-3}$ & 26 & $7.6 \times 10^{-6}$ & 0.254 & $9.3 \times 10^{-7}$ \\
\hline 9 & 10 & 23 & $1.2 \times 10^{-3}$ & 0.076 & $4.3 \times 10^{-4}$ & 27 & $8.1 \times 10^{-7}$ & 0.278 & $5.8 \times 10^{-7}$ \\
\hline 9 & 12 & 23 & $1.2 \times 10^{-3}$ & 0.074 & $5.5 \times 10^{-4}$ & 27 & $8.1 \times 10^{-7}$ & 0.271 & $6.3 \times 10^{-7}$ \\
\hline 9 & 14 & 23 & $1.2 \times 10^{-3}$ & 0.072 & $6.8 \times 10^{-4}$ & 27 & $8.1 \times 10^{-7}$ & 0.268 & $7.7 \times 10^{-7}$ \\
\hline 10 & 10 & 23 & $1.2 \times 10^{-3}$ & 0.074 & $5.1 \times 10^{-4}$ & 27 & $8.1 \times 10^{-7}$ & 0.242 & $6.4 \times 10^{-7}$ \\
\hline 10 & 12 & 23 & $1.2 \times 10^{-3}$ & 0.074 & $4.6 \times 10^{-4}$ & 27 & $8.1 \times 10^{-7}$ & 0.269 & $6.5 \times 10^{-7}$ \\
\hline 10 & 14 & 23 & $1.2 \times 10^{-3}$ & 0.070 & $7.3 \times 10^{-4}$ & 27 & $8.1 \times 10^{-7}$ & 0.234 & $9.8 \times 10^{-7}$ \\
\hline 11 & 10 & 23 & $1.2 \times 10^{-3}$ & 0.077 & $3.0 \times 10^{-4}$ & 27 & $8.1 \times 10^{-7}$ & 0.262 & $6.3 \times 10^{-7}$ \\
\hline 11 & 12 & 23 & $1.2 \times 10^{-3}$ & 0.074 & $3.8 \times 10^{-4}$ & 27 & $8.1 \times 10^{-7}$ & 0.252 & $8.8 \times 10^{-7}$ \\
\hline 11 & 14 & 23 & $1.2 \times 10^{-3}$ & 0.073 & $4.8 \times 10^{-4}$ & 27 & $8.1 \times 10^{-7}$ & 0.248 & $8.8 \times 10^{-7}$ \\
\hline 12 & 10 & 23 & $1.2 \times 10^{-3}$ & 0.077 & $2.6 \times 10^{-4}$ & 27 & $8.1 \times 10^{-7}$ & 0.257 & $7.0 \times 10^{-7}$ \\
\hline 12 & 12 & 23 & $1.2 \times 10^{-3}$ & 0.075 & $3.4 \times 10^{-4}$ & 27 & $8.1 \times 10^{-7}$ & 0.249 & $8.5 \times 10^{-7}$ \\
\hline 12 & 14 & 23 & $1.2 \times 10^{-3}$ & 0.073 & $4.3 \times 10^{-4}$ & 27 & $8.1 \times 10^{-7}$ & 0.236 & $1.0 \times 10^{-6}$ \\
\hline 20 & 20 & 23 & $1.2 \times 10^{-3}$ & 0.071 & $3.1 \times 10^{-4}$ & 25 & $5.2 \times 10^{-5}$ & 0.176 & $1.1 \times 10^{-5}$ \\
\hline
\end{tabular}
surrogates $\tilde{x}_{n}$ and $\tilde{y}_{n}$ coincide exactly with the original amplitudes.

\section{APPENDIX B}

TABLE I. Dependence of results on the embedding dimension $m$ and time delay $\tau_{D}$ used for the delay coordinates.

\footnotetext{
${ }^{\mathrm{a}}$ Number of cases of a total of 29 cases.

${ }^{\mathrm{b}}$ Note that the values of $p_{M}^{\mathrm{val}}$ do not directly follow from $\overline{\langle\Delta M\rangle}$. Rather, these values are derived from the distribution of all $\left\langle\Delta M{ }^{(v)}\right.$ values. Accordingly, higher (lower) values of $\overline{\langle\Delta M\rangle}$ are not necessarily reflected in lower (higher) values of $p_{M}^{\mathrm{val}}$.
} 
[1] V. L. Towle, I. Syed, C. Berger, R. Grzesczcuk, J. Milton, R. K. Erickson, P. Cogen, E. Berkson, and J. P. Spire, Electroencephalogr. Clin. Neurophysiol. 106, 30 (1998).

[2] H. R. Zaveri, S. M. Pincus, I. I. Goncharova, R. B. Duckrow, D. D. Spencer, and S. S. Spencer, NeuroReport 20, 891 (2009).

[3] H. Osterhage, F. Mormann, M. Staniek, and K. Lehnertz, Int. J. Bifurcation Chaos Appl. Sci. Eng. 17, 3539 (2007).

[4] C. Rummel, M. Müller, G. Baier, F. Amor, K. Schindler, J. Neuro. Methods. 191, 94 (2010).

[5] G. Bettus, F. Wendling, M. Guye, L. Valton, J. Regis, P. Chauvel, and F. Bartolomei, Epilepsy Res. 81, 58 (2008).

[6] J. Arnhold, K. Lehnertz, P. Grassberger, and C. E. Elger, Phys. D (Amsterdam) 134, 419 (1999).

[7] F. Mormann, K. Lehnertz, P. David, and C. E. Elger, Phys. D (Amsterdam) 144, 358 (2000).

[8] C. A. Schevon, J. Cappel, R. Emerson, J. Isler, P. Grieve, R. Goodman, G. Mckhann Jr., H. Weiner, W. Doyle, R. Kuzniecky, O. Devinsky, and F. Gilliam, Neuroimage 35, 140 (2007).

[9] E. Ben-Jacob, S. Boccaletti, A. Pomyalov, I. Procaccia, and V. L. Towle, Chaos 17, 043113 (2007).

[10] M. Paluš, V. Komárek, Z. Hrnčíř, and K. Štěrbová, Phys. Rev. E 63, 046211 (2001).

[11] M. Staniek and K. Lehnertz, Phys. Rev. Lett. 100, 158101 (2008).

[12] S. Sabesan, L. B. Good, K. S. Tsakalis, A. Spanias, D. M. Treiman, and L. D. Iasemidis, IEEE Trans. Neural. Syst. Rehab. Eng. 17, 244 (2009).

[13] J. Prusseit and K. Lehnertz, Phys. Rev. E 77, 041914 (2008).

[14] J. Theiler, S. Eubank, A. Longtin, B. Galdrikian, and J. D. Farmer, Phys. D (Amsterdam) 58, 77 (1992).

[15] D. T. Kaplan and R. J. Cohen, Circ. Res. 67, 886 (1990).

[16] J. P. Pijn, J. van Neerven, A. Noest, and F. Lopes da Silva, Clin. Neurophysiol. 79, 371 (1991).

[17] T. Schreiber and A. Schmitz, Phys. D (Amsterdam) 142, 346 (2000).

[18] R. G. Andrzejak, A. Kraskov, H. Stögbauer, F. Mormann, and T. Kreuz, Phys. Rev. E. 68, 066202 (2003).

[19] R. G. Andrzejak, F. Mormann, G. Widman, T. Kreuz, C. E. Elger, and K. Lehnertz, Epilepsy Res. 69, 30 (2006).

[20] R. G. Andrzejak, G. Widman, K. Lehnertz, P. David, and C. E. Elger, Epilepsy Res. 44, 129 (2001).
[21] M. C. Casdagli, L. D. Iasemides, R. S. Savit, R. L. Gilmore, S. N. Roper, and J. C. Sackellares, Electroencephalogr. Clin. Neurophysiol. 102, 98 (1997).

[22] A. Cenys, G. Lasiene, and K. Pyragas, Phys. D (Amsterdam) 52, 332 (1991).

[23] S. J. Schiff, P. So, T. Chang, R. E. Burke, and T. Sauer, Phys. Rev. E 54, 6708 (1996).

[24] R. Quian Quiroga, A. Kraskov, T. Kreuz, and P. Grassberger, Phys. Rev. E 65, 041903 (2002).

[25] R. Quian Quiroga, J. Arnhold, and P. Grassberger, Phys. Rev. E 61, 5142 (2000).

[26] H. Kantz and T. Schreiber, Nonlinear Time Series Analysis, 2nd ed. (Cambridge University, Cambridge, UK, 2003).

[27] M. C. Romano, M. Thiel, J. Kurths, and C. Grebogi, Phys. Rev. E 76, 036211 (2007).

[28] D. Chicharro and R. G. Andrzejak, Phys. Rev. E 80, 026217 (2009).

[29] F. Takens, Detecting Strange Attractors in Turbulence. Lecture Notes in Mathematics 898, 366, edited by D. A. Rand and L.-S. Young (Springer, Berlin, 1981).

[30] J. Theiler, Phys. Rev. A 34, 2427 (1986).

[31] D. A. Smirnov and R. G. Andrzejak, Phys. Rev. E 71, 036207 (2005).

[32] F. Mormann, T. Kreuz, C. Rieke, R. G. Andrzejak, A. Kraskov, P. David, C. E. Elger, and K. Lehnertz, Clin. Neurophysiol. 116, 569 (2005).

[33] F. Mormann, H. Osterhage, R. G. Andrzejak, B. Weber, G. Fernandez, J. Fell, C. E. Elger, and K. Lehnertz, Front. Hum. Neurosci. 2, 3 (2008).

[34] K. Lehnertz and C. E. Elger, Electroencephalogr. Clin. Neurophysiol. 103, 376 (1997).

[35] T. Schreiber and A. Schmitz, Phys. Rev. Lett. 77, 635 (1996).

[36] L. Faes, A. Porta, and G. Nollo, Phys. Rev. E 78, 026201 (2008).

[37] M. Thiel, M. C. Romano, J. Kurths, M. Rolfs, and R. Kliegl, Europhys. Lett. 75, 535 (2006).

[38] A. K. Seth, J. Neurosci. Methods 186, 262 (2010).

[39] K. Lehnertz, S. Bialonski, M. Horstmann, D. Krug, A. Rothkegel, M. Staniek, and T. Wagner, J. Neurosci. Methods 183, 42 (2009).

[40] D. Prichard and J. Theiler, Phys. Rev. Lett. 73, 951 (1994). 